\title{
Image-Guided Radiation therapy; Basic Concepts and Clinical Potentials
}

\author{
Omar Abdel-Rahman* \\ Clinical Oncology Department, Faculty of Medicine, Ain Shams University, Cairo, Egypt
}

*Corresponding author: Omar Abdel-Rahman, Clinical Oncology Department, Faculty of Medicine, Ain Shams University, Lotfy Elsayed Street, Cairo, Postal Code: 11665, Egypt, Tel: + 33028656; Fax: 33028656; E-mail: omar.abdelrhman@med.asu.edu.eg

Received date: Jun 07, 2014, Accepted date: Jul 07, 2014, Publication date: Jul 25, 2014

Copyright: () 2014 Abdel-Rahman O, This is an open-access article distributed under the terms of the Creative Commons Attribution License, which permits unrestricted use, distribution, and reproduction in any medium, provided the original author and source are credited.

\begin{abstract}
The adaptation and integration of imaging into the process of cancer detection, diagnosis, and intervention is an area of medicine that is undergoing extremely rapid development. Radiation therapy is a prime example of this change. While the objectives of these developments are clear, they raise numerous issues regarding the skills and resources that assure these technologies are appropriately integrated and applied. We will explore the basic concepts related to image guidance in various radiotherapy-related procedures with special emphasis on the clinical potentials of this impressive technology.
\end{abstract}

Keywords: Image guidance; Radiation therapy

\section{Overview}

The adaptation and integration of imaging into the process of cancer detection, diagnosis, and intervention is an area of medicine that is undergoing extremely rapid development. Radiation therapy is a prime example of this change. As a result, imaging is now a pervasive component of radiation therapy with all major imaging modalities represented and numerous examples in which these modalities have been used in treatment planning to allow increased accuracy and precision in the delivery of dose [1]. While the objectives of these developments are clear, they raise numerous issues regarding the skills and resources that assure these technologies are appropriately integrated and applied. Specifically, these developments place enormous pressure on the clinical staff to extend their knowledge base and their scope of responsibility [2].

The term IGRT is defined as " the use of modern imaging modalities, especially those incorporating functional or biological information, to augment target delineation, and the use of imaging to adjust for target motion or positional uncertainty, and, potentially, to adapt treatment to tumour response" [3].

IGRT has the potential to target gross and microscopic disease accurately, to individualize treatments to reduce margins and to allow radiation dose escalation to higher levels with the expectation of improving local control and reducing toxicity. Although this may be the case in principle, and there is some anecdotal and indirect evidence to support this assertion, continuing research to further develop the IGRT methods and their application in clinical trials are required to determine the true clinical promise of IGRT [4].

\section{Rationale of IGRT}

Shifting patterns of clinical presentation of cancer and advances in local and systemic therapies altering patterns of failure make optimal target definition a constant, evolving challenge. Modern imaging modalities assist in this regard by improving disease detection and refining target definition, thus optimizing therapeutic results. One rationale of IGRT is that reproducibly positioning the patient is an important element of fractionated RT delivery. However, both systematic and stochastic errors in patient setup contribute to variation in daily positioning. Reproducibility is also hindered by movement or changes occurring in target and normal tissues between (interfraction) or even during (intrafraction) treatment. IGRT incorporates strategies to more accurately localize the target and optimize RT during the treatment course, ideally leading to more effective and less toxic therapy $[3,5]$.

\section{Search Strategy}

I conducted an independent review of Medline databases from January 1966 to September 2013 using "image-guided radiation therapy" OR "IGRT" as a search keyword. The search was limited to human, cancer, and clinical trials published in English.

\section{Clinical Application}

\section{Image-guided target definition}

Positron emission tomography: At most centres, CT-based planning is now commonplace and its impact on improved target delineation are well known. Interest is now focused on incorporation of more sophisticated imaging approaches, particularly those that provide functional and biologic information. The most frequently studied of these techniques is PET scan [6].

PET is currently accepted as an important tool in oncology, mostly for diagnosis, staging and restaging purposes. It provides a new type of information in radiotherapy, functional rather than anatomical. PET imaging can also be used for target volume definition in radiotherapy treatment planning [7].

As regard target definition and altered dose distribution, PET has already been shown to be valuable for defining the extent of target volumes. Furthermore, PET can be used to differentiate areas of biological importance within the boundaries of target volumes $[6,8]$.

Studies of ${ }^{18}$ FDG-PET in head and neck cancer have found promising results, with RT volumes frequently altered after incorporating PET imaging [9]. In a study of 40 head and neck cancer 
patients, one of these studies has compared IMRT plans based on ${ }^{18}$ FDG-PET to those based on CT. In $25 \%$ of patients, CT-based plans were suboptimal in covering the PET-delineated GTV [10].

Multiple studies have investigated the role of ${ }^{18} \mathrm{FDG}$-PET in RT planning for non-small cell lung cancer (NSCLC) [11]. One of these studies found that ${ }^{18} \mathrm{FDG}$-PET-CT altered treatment volumes in $45 / 73$ (62\%) lymph node positive patients staged by mediastinoscopy; in 16 the volume was enlarged, and in 29 it was contracted [12]. While another study confirmed the utility of ${ }^{18}$ FDG-PET in NSCLC treatment planning, noting significant changes in the gross tumour volume (GTV) and planning target volumes (PTV) in $58 \%$ of patients [13].

Another potential role for PET is in 3D brachytherapy planning, Lin and coworkers described previously FDG-PET based 3D brachytherapy treatment planning for treatment of cervical cancer. In this study it was demonstrated that FDG-PET based treatment planning allowed for improved dose coverage of the tumour without significantly increasing the dose to the bladder and rectum [14].

Magnetic resonance imaging (MRI): MRI-based treatment planning was studied in a number of tumour sites; most notably intracranial and pelvic tumours [15]. For intracranial tumours, one study has reached the conclusion that MRI-based treatment planning procedure is suitable for the radiotherapy of intracranial lesions [16].

Moreover, MRI was studied in MRI-Guided Intracavitary Brachytherapy for Cancer of the Cervix. In one study, a total of 20 patients with International Federation of Gynecology and Obstetrics Stage IB-IV cervical cancer had an MRI-compatible intrauterine BT applicator inserted after external beam radiotherapy. It was then concluded that MRI-based BT for cervical cancer has the potential to optimize primary tumour dosimetry and reduce the dose to critical normal tissues, particularly in patients with small tumours [17].

Single Photon Emission Computed Tomography (SPECT): SPECT technology has also been explored in radiation therapy planning for many tumour sites [18]; one study has previously reported that RIS (radioimmunoscintigraphy) influenced RT volumes and decision making in a significant proportion of patients undergoing post prostatectomy salvage RT. Of 54 evaluable patients, $18.5 \%$ had treatment plans altered by RIS, including four who were not offered RT based on the RIS findings [19].

\section{Correction of motion and setup errors through IGRT technologies}

2D Radiographic imaging: Two-dimensional (2-D) radiographic imaging is typically used in treatment rooms to align the patient relative to the radiation beams. Megavoltage electronic portal image (EPI) is the classic example. Megavoltage (MV) imaging, including the traditional $\mathrm{x}$-ray films, uses therapy $\mathrm{x}$-ray beams to verify the patient's setup positions [20]. In addition, MV imaging is also used to verify the shape of the treatment portals. Because the same therapy high-energy $\mathrm{x}$-ray beam is used for verification, it represents a method for direct infield verification of treatment delivery. Disadvantages of the pretreatment MV imaging include high imaging dose (typically 1 to 5 cGy) and poor image quality due to the higher $\mathrm{x}$-ray beam energies [4].

Several investigators have reviewed their experience using online EPID (electronic portal imaging device) IGRT approaches. In 16 inoperable lung cancer patients, the locations of the lung apices and the carina were used for alignment. In-house software was used to calculate translational and rotational errors. If either exceeded $2 \mathrm{~mm}$, a correction was performed, resulting in corrections in $85 \%$ of setups [21].

Considerable attention has also been focused on EPID as a means of prostate localization in conjunction with implanted seed markers. One study has reported the experience of using EPID and implanted prostate seed markers in 17 patients. Using an action level of $3 \mathrm{~mm}$, $19 \%$ of the treatment fractions were adjusted, requiring, on average, 6.1 additional minutes when no adjustments were required and 8.7 minutes when adjustments were made [22].

More recently, other forms of 2-D imaging have been introduced. An example is the kilovoltage $(\mathrm{kV}) \mathrm{x}$-ray tube combined with a flatpanel image detector mounted orthogonal to the therapy $\mathrm{x}$-ray beam on a linear accelerator. Such on-board imagers (OBIs) provide diagnostic quality $\mathrm{x}$-ray images to setup the patient by alignment with the reference digitally reconstructed radiographs (DRRs) computed from the treatment planning systems. Although the contrast of $\mathrm{kV}$ static $\mathrm{x}$-rays is considerably superior compared to MV EPIs, it is often not sufficient for detecting soft tissue targets [4]. OBIs are more successful at aligning bony landmarks or implanted radio-opaque markers (fiducials) as surrogates of the soft tissue target. In-room $\mathrm{kV}$ $\mathrm{x}$-ray imaging represents a major upgrade from the traditional electronic portal imaging devices (EPIDs) due to its superior image quality to visualize bony structures and its low imaging dose. In addition, $\mathrm{kV} \mathrm{x}$-ray images resemble the look of traditional simulation images and DRRs calculated to enhance bone contrast [23].

3D volumetric imaging: 3-D "volumetric" imaging inside a treatment room represents the latest development in the IGRT armamentariums. With the patient immobilized on the treatment couch, true 3-D (voxel-by-voxel) information can be acquired with a CT scanner in the same room just before the start of treatment, which allows for more accurate guidance to setup the patient's position relative to the treatment beams. In the following subsections, we describe various forms of in-room CT implementations based on $\mathrm{x}$-ray energies used ( $\mathrm{kV}$ vs. $\mathrm{MV}$ ) and detector configurations (helical slit geometry vs. large-field cone-beam geometry) [24].

KV Computed tomography: Helical single or multiple-slice CT systems have been widely used in diagnostic imaging and routine treatment planning for many years. For CT-guided radiotherapy applications, the first integrated clinical system combining a linear accelerator (linac) and an in-treatment-room conventional CT unit was developed by Uematsu and coworkers at the National Defense Medical College, Saitama, Japan [25].

The outcome of 35 paraspinal tumor patients (14 primary, 21 metastatic) undergoing IMRT using such a system at the MSKCC has been presented. Overall, 24 (68\%) had received prior RT. A planning margin of $10 \mathrm{~mm}$ was used, except at the spinal cord interface where 5 $\mathrm{mm}$ was used. The median prescribed dose was $2000 \mathrm{cGy}$ in five fractions. At a median follow-up of 11 months, the 2-year actuarial local control rates for primary and metastatic tumors were $75 \%$ and $81 \%$, respectively [26].

\section{Tomotherapy (Helical Megavoltage Computed Tomography)}

Tomotherapy is an integrated technology that combines a helical megavoltage CT (MVCT) with a linear accelerator which is specially designed for delivering intensity-modulated radiation in a slit geometry. Helical tomotherapy refers to the continuous gantry and 
couch motion, which resembles the motion from a conventional helical CT scanner. Low dose, typically 1 to $2 \mathrm{cGy}$, pretreatment MVCT images can be reconstructed from the same MV x-ray beam it uses for treatment [27].

One study has reported on the use of the Tomotherapy system for optimizing patient setup in eight patients undergoing reirradiation of spinal metastases. The mean retreatment dose was $28 \mathrm{~Gy}$, with the maximum cord dose of $27 \%$ to $56 \%$ of the prescribed dose. None developed an in-field recurrence or significant late toxicity [28].

In another study, application of helical tomotherapy $(\mathrm{HT})$ in whole skull palliative radiotherapy was tested. An inverse HT plan and an accompanying back-up conventional lateral 6-MV parallel opposed pair (POP) plan with corresponding isodose distributions and dosevolume histograms (DVH) were created and assessed prior to initiation of therapy. Conformal sparing of brain, optic nerve, and eye was achieved by the HT plan. However, similar lens and brain stem/ spinal cord doses were seen with both plans [29].

\section{Megavoltage Cone-beam Computed Tomography}

Megavoltage cone-beam CT (MVCBCT) uses the traditional EPID mounted on a linac gantry and the therapy $\mathrm{MV} \mathrm{x}$-ray as a basic configuration for a CT imaging system. An evaluation of the potential benefits of MVCBCT imaging in head and neck, lung, and pelvic patients treated on a prospective clinical trial was done. In a locally advanced head and neck cancer patient, MVCBCT detected a misalignment of the vertebral bodies and spinal cord in the neck not seen on portal imaging [30].

In another study, Refinement of treatment setup and target localization accuracy using three-dimensional cone-beam computed tomography for stereotactic body radiotherapy was studied. After localization based on superficial markings in patients undergoing SBRT, orthogonal $\mathrm{kV}$ imaging detects setup variations of approximately 3 to $4 \mathrm{~mm}$ in each direction. Whereas cone-beam CT detects residual setup variations of approximately 2 to $3 \mathrm{~mm}$ [31].

\section{KV Cone-beam Computed Tomography}

A $\mathrm{kV}$ imaging system capable of radiography, fluoroscopy, and cone-beam CT would be an ideal solution for IGRT applications. Recent effort in integrating such a system with a medical linear accelerator has produced enormous interest in these systems. One study has compared the utility of the $\mathrm{kV} \mathrm{CBCT} \mathrm{system} \mathrm{with} \mathrm{EPID} \mathrm{in}$ terms of setup accuracy in 24 patients with a variety of tumours. Kilovoltage CBCT was found to add little in the assessment of translational errors. Translational errors detected with either approach differed by $<1 \mathrm{~mm}$ in $70.7 \%$ and $<2 \mathrm{~mm}$ in $93.2 \%$ of measurements. However, СBCT was superior in the detection of rotational errors. Rotational errors $>2$ degrees were noted in $3.7 \%, 26.4 \%$, and $12.4 \%$ of pelvic, thoracic, and head and neck tumours, respectively. Such rotational errors led to poorer target coverage and increased normal tissue dose in cases with elongated targets in close proximity to normal tissues [32].

\section{Ultrasound Systems}

Through the use of high-frequency sound waves to produce images of internal anatomy, ultrasound is one of the most common IGRT approaches in current practice. Although three operational modes are available, B (brightness) mode is the primary one used. The most common ultrasound system in current clinical use is the B-mode acquisition and targeting (BAT) transabdominal system, consisting of a probe and a computer-based targeting system. The probe is registered to a stereotactic arm on the linac gantry, allowing its position to be tracked [33].

One study has evaluated BAT system in 147 prostate IMRT patients and reported interfraction standard deviations of prostate position of $4.9,4.4$, and $2.8 \mathrm{~mm}$ in the anteroposterior (AP), superior-inferior (SI) and lateral (RL) dimensions, respectively. The percentage of shifts $>5$ $\mathrm{mm}$ was $28.6 \%$ in $\mathrm{AP}, 23 \%$ in SI, and $9 \%$ in RL, respectively [34].

\section{Use of IGRT in respiratory motion management}

Respiratory-induced organ motion is a major problem in RT, particularly in tumors in the thorax and upper abdomen. Tumours in these sites can move up to $2.5 \mathrm{~cm}$ or more during free breathing. Such motions result in imaging artifacts and positional uncertainty that compromise the accuracy of target delineation and treatment delivery [35]. A common method to account for uncertainties in targeting has been to use standard, population-based, PTV margins, typically 5 to 15 $\mathrm{mm}$. However, this approach can result in irradiation of large volumes of normal tissues in some patients, while under dosing the target in others [36]. Customized margins have been advocated based on the maximum excursion of tumour observed during fluoroscopy. Others have explored the use of breath-hold techniques that "freeze" the target in a specific phase of the respiratory cycle, notably $A B C$ and deep inspiration breath hold (DIBH). However, such approaches may not be well tolerated in many patients, particularly those with poor pulmonary function. More sophisticated methods to improve target delineation and treatment of mobile tumours are respiratory gating and $4 \mathrm{D}$ imaging [4].

Respiratory gating: The two main approaches to respiratory gating are internal and external gating. Internal gating utilizes internal tumour motion surrogates such as implanted fiducial markers, whereas external gating uses external respiratory surrogates such as markers placed on the surface of the patient's abdomen [37].

4D imaging: 4D imaging combines the spatial (e.g., 3D) data with time-dependent changes during respiration. And this section will concentrate on $4 \mathrm{D} \mathrm{CT}$. The idea behind $4 \mathrm{D}$ CT is that, at every position of interest along patient's long axis, images are oversampled and each image is tagged with breathing phase information. After the scan is done, images are sorted based on the corresponding breathing phase signals. Many 3D CT sets are thus obtained, each corresponding to a particular breathing phase, and together they constitute a $4 \mathrm{D}$ CT set covering the whole breathing cycle [38].

4D Target delineation: The approaches to $4 \mathrm{D}$ target delineation enable construction of an integrated target volume (ITV) consisting of imaging data acquired in separate phases of respiration into a combined $3 \mathrm{D}$ volume containing the probable location of tumour. For example, Allen and co-workers created a composite volume based on the tumour delineated on maximal inhalation and exhalation scans in 16 patients. This structure was significantly smaller than a $1 \mathrm{~cm}$ uniform expansion around the GTV delineated on a free-breathing scan, indicating that a standard approach using a $1-\mathrm{cm}$ expansion leads to overtreatment of normal tissues [39]. 


\section{Use of IGRT technologies in Adaptive Radiotherapy}

Adaptive radiotherapy is a treatment technique that can systematically improve its treatment plan in response to patient/organ temporal variations observed during the therapy process. Temporal variations in radiotherapy process can be either patient/organ geometry or dose-response related. Examples of the former include inter- and intra-treatment variations of patient/organ shape and positions caused by patient setup, beam placement, and patient organ physiological motion and deformation. Examples of dose-response characteristics include the variations of size and location of tumour hypoxic volume, the apparent tumour growth fraction, and normal tissue damage/repair kinetics. Furthermore, tumour and normal organ dose response also induce changes in tissue shape and positions [40].

Adaptive RT (ART) is a broad concept. One approach is to use the knowledge of patient setup and organ motion obtained from imaging during treatment to alter the treatment plan. This approach was introduced by investigators at William Beaumont Hospital in patients with prostate cancer. As initially conceived, treatment is initiated with a population-based margin and setup errors are measured daily using EPID imaging. Systematic and random setup errors and the timedependent drift of treatment setup are then predicted [41].

For example, Vargas and co-workers treated a total of 331 T1-3 prostate patients using the Beaumont adaptive process. At a median follow-up of 1.6 years, grade 2 chronic rectal toxicity was seen in $10 \%$ of patients; nine (3\%) developed grade 3 chronic rectal toxicity. The 2 year actuarial rate of grade 2 and 3 rectal chronic sequelae was $17 \%$ and $3 \%$, respectively. No difference was noted between dose levels, supporting the adaptive process [42].

Another adaptive approach is to alter treatment based on changes in the tumour and/or normal tissues. For example, Van de Bunt and co-workers reported an average reduction in the GTV of $46 \%$ in 14 cervical cancers reimaged with MRI after the delivery of $30 \mathrm{~Gy}$ [43].

\section{Conclusions}

The use IGRT technologies have changed the way of radiation therapy planning and delivery for many tumour sites. However, IGRT may be associated with significant increases in cost to patients and the medical system, and economic competition is likely to have an important impact on its implementation in general radiation oncology practice. Therefore, continued careful study and quantification of the effects of IGRT on treatment outcomes are needed so that definitive statements may be made about its necessity in everyday radiotherapy and for which tumour sites.

\section{References}

1. http://www-naweb.iaea.org/nahu/DMRP/documents/ IAEA_Report_Imaging_in_RT.pdf

2. Ahmad SS1, Duke S, Jena R, Williams MV, Burnet NG (2012) Advances in radiotherapy. BMJ 345: e7765.

3. Mell L, Pawlicki T, Jiang S (2007) Image-Guided Radiation Therapy: Principles and Practice of Radiation Oncology. Lippincott Williams \& Wilkins: 5.

4. Dong L, Mohan R (2007) Image-Guided Radiation Therapy: Treatment Planning in Radiation Oncology. Lippincott Williams \& Wilkins: 2.

5. Jaffray DA (2012) Image-guided radiotherapy: from current concept to future perspectives. Nat Rev Clin Oncol 9: 688-699.
6. Mutic S (2006) PET and PET/CT for Radiotherapy Planning: New Technologies in Radiation oncology. Springer-Verlag Berlin Heidelberg: 1.

7. Rembielak A, Price P (2008) The role of PET in target localization for radiotherapy treatment planning. Onkologie 31: 57-62.

8. Götz L, Spehl TS, Weber WA, Grosu AL (2012) PET and SPECT for radiation treatment planning. Q J Nucl Med Mol Imaging 56: 163-172.

9. Garg MK, Glanzman J, Kalnicki S (2012) The evolving role of positron emission tomography-computed tomography in organ-preserving treatment of head and neck cancer. Semin Nucl Med 42: 320-327.

10. Paulino AC, Koshy M, Howell R, Schuster D, Davis LW (2005) Comparison of CT- and FDG-PET-defined gross tumor volume in intensity-modulated radiotherapy for head-and-neck cancer. International Journal of Radiation Oncology. Biology and Physics 61: 1385-1392.

11. Belderbos J, Sonke JJ (2009) State-of-the-art lung cancer radiation therapy. Expert Rev Anticancer Ther 9: 1353-1363.

12. Vanuytsel LJ, Vansteenkiste JF, Stroobants SG, De Leyn PR, De Wever W, et al. (2000) The impact of (18)F-fluoro-2-deoxy-D-glucose positron emission tomography (FDG-PET) lymph node staging on the radiation treatment volumes in patients with non-small cell lung cancer. Radiother Oncol 55: 317-324.

13. Bradley J, Thorstad WL, Mutic S, Miller TR, Dehdashti F, et al. (2004) Impact of FDG-PET on radiation therapy volume delineation in nonsmall-cell lung cancer. Int J Radiat Oncol Biol Phys 59: 78-86.

14. Lin LL, Mutic S, Low DA, LaForest R, Vicic M, et al. (2007) Adaptive brachytherapy treatment planning for cervical cancer using FDG-PET. Int J Radiat Oncol Biol Phys 67: 91-96.

15. Stanescu T, Jans HS, Pervez N, Stavrev P, Fallone BG (2008) A study on the magnetic resonance imaging (MRI)-based radiation treatment planning of intracranial lesions. Phys Med Biol 53: 3579-3593.

16. Devic S (2012) MRI simulation for radiotherapy treatment planning. Med Phys 39: 6701-6711.

17. Zwahlen D, Jezioranski J, Chan P, Haider MA, Cho YB, et al. (2009) Magnetic resonance imaging-guided intracavitary brachytherapy for cancer of the cervix. Int J Radiat Oncol Biol Phys 74: 1157-1164.

18. Jani AB, Blend MJ, Hamilton R, Brendler C, Pelizzari C, et al. (2004) Influence of radioimmunoscintigraphy on postprostatectomy radiotherapy treatment decision making. J Nucl Med 45: 571-578.

19. Apisarnthanarax S, Chao KS (2005) Current imaging paradigms in radiation oncology. Radiat Res 163: 1-25.

20. Michalski A, Atyeo J, Cox J, Rinks M (2012) Inter- and intra-fraction motion during radiation therapy to the whole breast in the supine position: a systematic review. J Med Imaging Radiat Oncol 56: 499-509.

21. Van de Steene J, Van den Heuvel F, Bel A, Verellen D, De Mey J, et al. (1998) Electronic portal imaging with on-line correction of setup error in thoracic irradiation: clinical evaluation. International Journal of Radiation Oncology, Biology and Physics 40: 967-976.

22. Chung PWM, Haycocks T, Brown T, Cambridge Z, Kelly V, et al. (2004) On-line aSi portal imaging of implanted fiducial markers for the reduction of interfraction error during conformal radiotherapy of prostate carcinoma. International Journal of Radiation Oncology. Biology and Physics 60: 329-334.

23. Guckenberger M, Richter A, Boda-Heggemann J, Lohr F (2012) Motion compensation in radiotherapy. Crit Rev Biomed Eng 40: 187-197.

24. Li G, Citrin D, Camphausen K, Mueller B, Burman C, et al. (2008) Advances in $4 \mathrm{D}$ medical imaging and $4 \mathrm{D}$ radiation therapy. Technol Cancer Res Treat 7: 67-81.

25. Uematsu M, Fukui T, Shioda A, Tokumitsu H, Takai K, et al. (1996) A dual computed tomography linear accelerator unit for stereotactic radiation therapy: a new approach without cranially fixated stereotactic frames. International Journal of Radiation Oncology. Biology and Physics 35: $587-592$. 
Citation: Abdel-Rahman O (2014) Image-Guided Radiation therapy; Basic Concepts and Clinical Potentials. J Nucl Med Radiat Ther 5: 181. doi: $10.4172 / 2155-9619.1000181$

Page 5 of 5

26. Wright JL, Lovelock DM, Bilsky MH, Toner S, Zatcky J, et al. (2006) Clinical outcomes after reirradiation of paraspinal tumors. Am J Clin Oncol 29: 495-502.

27. Zhang L, Chen QY, Liu H, Tang LQ, Mai HQ (2013) Emerging treatment options for nasopharyngeal carcinoma. Drug Des Devel Ther 7: 37-52.

28. Mohan R, Zhang X, Wang H, Kang Y, Wang X, et al. (2005) Use of deformed intensity distributions for on-line modification of imageguided IMRT to account for interfractional anatomic changes. International Journal of Radiation Oncology. Biology and Physics 61: 1258-1266.

29. Rodrigues G, Yartsev S, Coad T, Bauman G (2008) Novel application of helical tomotherapy in whole skull palliative radiotherapy. Medical Dosimetry 33: 282-5.

30. Morin O, Gillis A, Chen J, Aubin M, Bucci MK, et al. (2006) Megavoltage cone-beam CT: system description and clinical applications. Med Dosim 31: 51-61.

31. Wang Z, Nelson JW, Yoo S, Jackie Wu Q, P. Kirkpatrick J, et al. (2009) Refinement of treatment setup and target localization accuracy using three-dimensional cone-beam computed tomography for stereotactic body radiotherapy. International Journal of Radiation Oncology. Biology and Physics 73: 571-7.

32. Guckenberger M, Meyer J, Vordermark D, Baier K, Wilbert J, et al. (2006) Magnitude and clinical relevance of translational and rotational patient setup errors: a cone-beam CT study. Int J Radiat Oncol Biol Phys 65: 934-942.

33. Wu VW, Law MY, Star-Lack J, Cheung FW, Ling CC (2011) Technologies of image guidance and the development of advanced linear accelerator systems for radiotherapy. Front Radiat Ther Oncol 43: 132-164.

34. Chandra A, Dong L, Huang E, Kuban DA, O'Neill L, et al. (2003) Experience of ultrasound-based daily prostate localization. Int J Radiat Oncol Biol Phys 56: 436-447.
35. Bettinardi V, Picchio M, Di Muzio N, Gilardi MC (2012) Motion management in positron emission tomography/computed tomography for radiation treatment planning. Semin Nucl Med 42: 289-307.

36. Jiang SB (2006) Radiotherapy of mobile tumors. Semin Radiat Oncol 16: 239-248.

37. Korreman SS, Juhler-Nøttrup T, Persson GF, Navrsted Pedersen A, Enmark M, et al. (2008) The role of image guidance in respiratory gated radiotherapy. Acta Oncol 47: 1390-1396.

38. Moorrees J, Bezak E (2012) Four dimensional radiotherapy: a review of current technologies and modalities. Australas Phys Eng Sci Med 35: $399-406$.

39. Allen AM, Siracuse KM, Hayman JA, Balter JM (2004) Evaluation of the influence of breathing on the movement and modeling of lung tumors. Int J Radiat Oncol Biol Phys 58: 1251-1257.

40. Yan D, Wong JW, Gustafson G, Martinez A (1995) A new model for "accept or reject" strategies in off-line and on-line megavoltage treatment evaluation. Int J Radiat Oncol Biol Phys 31: 943-952.

41. Yan D (2006) Image-Guided/Adaptive Radiotherapy: New Technologies in Radiation oncology. Springer-Verlag Berlin Heidelberg: 1.

42. Vargas C, Yan D, Kestin LL, Krauss D, Lockman DM, et al. (2005) Phase II dose escalation study of image-guided adaptive radiotherapy for prostate cancer: use of dose-volume constraints to achieve rectal isotoxicity. Int J Radiat Oncol Biol Phys 63: 141-149.

43. Van de Bunt L, van der Heided UA, Ketelaars M, de Kort GA, Jürgenliemk-Schulz IM (2006) Conventional, conformal and intensity modulated radiation therapy treatment planning of external beam radiotherapy for cervical cancer: the impact of tumor regression. International Journal of Radiation Oncology, Biology and Physics 64: 189-196.
This article was originally published in a special issue, entitled: "Cancer Radiation Therapy", Edited by Xin Chen,University of Arkansas for Medical Sciences, USA 